\title{
Method of stimulation, mouth movements, concentration, and viscosity: Effects on the degree of taste adaptation
}

\author{
MARJON J. M. THEUNISSEN \\ Utrecht University, Utrecht, The Netherlands \\ JAN H. A. KROEZE \\ Utrecht University, Utrecht, The Netherlands \\ and The Wageningen Taste and Smell Center, Wageningen, The Netherlands \\ and \\ HENDRIK N. J. SCHIFFERSTEIN \\ Wageningen University, Wageningen, The Netherlands
}

\begin{abstract}
Although sensory adaptation, the gradual loss of sensation during prolonged stimulation, has been demonstrated in laboratory taste experiments, a comparable loss of taste intensity is not experienced in real-life eating situations. This discrepancy may be due to differences in the proximal stimuli or to differences in the ways the taste receptors are stimulated. In two experiments, the effects of four potentially relevant variables were investigated: stimulus intensity, stimulus viscosity, mouth movements, and presentation method. During the initial seconds of stimulation, adaptation to the weakest of the two solutions was faster. Although more viscous stimuli were less sweet, viscosity as such did not affect adaptation rate, nor did mouth movements. Among the three presentation methods, a sucrosesoaked filter paper on the tongue produced more adaptation than either sipping the solution or flowing it over the tongue. This suggests that even mouth movements far more subtle than those still present in the no-movement condition of a sip-and-spit experiment can disrupt the adaptation process.
\end{abstract}

Just like the other senses, the sense of taste exhibits sensory adaptation-that is, a gradual loss of sensation during prolonged stimulation (McBurney, 1985). Taste adaptation has been demonstrated in behavioral (e.g., Gent \& McBurney, 1978; Hahn, 1934; Meiselman, 1968) as well as in electrophysiological experiments (e.g., Smith, Bealer, \& Van Buskirk, 1978). Furthermore, Zotterman (1971) has demonstrated that the psychophysical and the electrophysiological time courses of taste adaptation match closely in human subjects. Surprisingly, this phenomenon, which is very robust in laboratory studies, seems to play hardly any role in our daily lives. During meals, taste does not disappear, not even during a single bite or sip. In order to understand why taste adaptation occurs in the laboratory and not in daily life, the variables that either enhance or counteract taste adaptation must be investigated. The present study is an attempt to do so with respect to four variables: mouth movements, method of stimulation, stimulus concentration, and stimulus viscosity.

This work was supported by Grant 575-62-057 from the Netherlands Organization for Scientific Research to J.H.A.K. Correspondence concerning this article should be addressed to M. J. M. Theunissen, Psychological Laboratory, Psychonomics Department, Utrecht University, Heidelberglaan 2,3584 CS Utrecht, The Netherlands (e-mail: m.theunissen@ fss.uu.nl).
Mouth movements are likely to affect the degree of adaptation, because they interfere with the continuity of stimulation, which probably is a necessary condition for adaptation (Gent \& McBurney, 1978; O’Mahony, 1986). By mouth movements made during eating, stimulus material is continuously moved over the tongue surface. As a consequence, previously stimulated and, thus, adapted parts of the tongue may recover from adaptation, while new areas are stimulated (O’Mahony \& Wong, 1989; Theunissen \& Kroeze, 1996; Von Békésy, 1965). In this way, mouth and tongue movements may be instrumental in maintaining perceived taste intensity by allowing recovery from adaptation. Meiselman and co-workers found that individual differences in degrees of adaptation were partly due to differences in the ability of individuals to keep the mouth still (DuBose, Meiselman, Hunt, \& Waterman, 1977; Meiselman \& Buffington, 1980).

The stimulation method may also affect the continuity of the stimulus. Three methods are generally used: sipand-spit, gravitational flow, and filter paper. When filter paper is used, a very constant stimulus is obtained, because the solution cannot move to another part of the tongue, even if mouth movements are made. A gravitational flow method is expected to give less opportunity for mouth movements than does a sip-and-spit method, depending on whether a tongue fixation apparatus or a chinrest is 
used. With a tongue fixation apparatus, hardly any tongue movements are possible. With a chinrest, some movement may occur, and if no chinrest is used, the freedom of movement is even larger. Table 1 shows the results from a number of studies in which the time course of adaptation was investigated, using different stimulation methods. In order to facilitate comparison, the degree of adaptation was computed as the percentage of decline of reported intensity over a period of $60 \mathrm{sec}$. There are large differences within and between studies, but the degree of adaptation appears to be slightly higher in the filter paper studies.

The discrepancy between adaptation during the eating of real foods and during laboratory experiments in which aqueous solutions of tastants are employed may also be related to differences in stimulus characteristics. In several studies, a higher degree of adaptation was observed for low concentrations than for high concentrations (DuBose et al., 1977; Gent, 1979; Lawless \& Skinner, 1979; Schiffman et al., 1994). Also, Gent observed that the stimulation of small tongue areas yielded a higher degree of adaptation than did that of larger areas. She proposed that the degree of adaptation is related to perceived intensity, rather than to concentration. As viscosity has usually been found to diminish the initial sweetness intensity of sucrose solutions (e.g., Christensen, 1980), higher viscosity levels could be expected to show an increased degree of adaptation.

\section{EXPERIMENT 1}

In this experiment, the combined effects of viscosity, sucrose concentration, and rate of mouth movements were examined for group and individual data. Both low concentrations and high viscosity levels were expected to lead to more adaptation than would high concentrations and low viscosity levels, respectively. We expected less adaptation when movements were made and even less adaptation when the subjects were free to choose their own rate of mouth movements. The latter prediction was based on the assumption that subjects try to counteract the effects of adaptation by mouth movements.

\section{Method}

Subjects. Twenty-one paid volunteers, 14 females and 7 males, ranging in age from 18 to 24 years (median, 21 years), served as subjects. They were students at Utrecht University. Some had experience with psychophysical experiments, but all were naive with respect to the purpose of the present experiment.

Stimuli. The stimuli were two concentrations of sucrose $(0.28$ and $0.56 \mathrm{M})$ at three viscosity levels $(1,1,400$, and $4,200 \mathrm{mPa} \cdot \mathrm{s})$. Sucrose (commercial grade, Suiker unie, Breda) was dissolved in demineralized water (produced by a Millipore Milli-U10 water purification system; resistivity $>10 \mathrm{M} \Omega \cdot \mathrm{cm}$ ). As a thickener, carboxymethylcellulose sodium salt was used (CMC; Fluka Chemica, 21904, ultra high viscosity). The amounts of CMC required to thicken the solutions were $0.08 \%(\mathrm{w} / \mathrm{w})$ and $1.26 \%$ for the $0.28 \mathrm{M}$ solutions and $0.71 \%$ and $1.16 \%$ for the $0.56 \mathrm{M}$ solutions. Viscosity was measured with a Brookfield viscometer (model LVF, spindle 3 at 60

Table 1

Degree of Taste Adaptation (as a Percentage) After 60 Sec for Different Methods of Stimulation

\begin{tabular}{|c|c|c|c|}
\hline Study & Method of Stimulation & Tastant & $\begin{array}{r}\text { Degree of } \\
\text { Adaptation }\end{array}$ \\
\hline \multirow[t]{3}{*}{ Meiselman (1968) } & sip-and-spit, gentle tongue movements & $\mathrm{NaCl}$ & $\sim 55-80$ \\
\hline & & quinine sulfate & $\sim 40-65$ \\
\hline & & sucrose & $\sim 55-65$ \\
\hline \multirow[t]{2}{*}{ O'Mahony and Wong (1989) } & sip-and-spit, chewing movements & $\mathrm{NaCl}$ & $\sim 37$ \\
\hline & sip-and-spit, motionless mouth & $\mathrm{NaCl}$ & $\sim 54$ \\
\hline Lawless and Skinner (1979) & dorsal flow & sucrose & $\sim 90-100$ \\
\hline Bujas, Szabo, Ajdukovic, and Mayer (1991) & dorsal flow, flow chamber & $\mathrm{NaCl}$ & $\sim 75$ \\
\hline DuBose and Meiselman (1979); & dorsal flow, tongue fixation & $\mathrm{NaCl}$ & $\sim 10-60$ \\
\hline DuBose, Meiselman, Hunt, and Waterman (1977) & apparatus & sucrose & $-20-55$ \\
\hline \multirow[t]{2}{*}{ Meiselman and Buffington (1980) } & $\begin{array}{l}\text { dorsal flow, tongue fixation } \\
\text { apparatus }\end{array}$ & $\mathrm{NaCl}$ & $\sim 25$ \\
\hline & filter paper & $\mathrm{NaCl}$ & $\sim 50$ \\
\hline \multirow[t]{4}{*}{ Ganzevles and Kroeze (1987) } & filter paper & $\mathrm{NaCl}$ & $\sim 50$ \\
\hline & & citric acid & $\sim 60$ \\
\hline & & quinine sulfate & $\sim 55$ \\
\hline & & sucrose & $\sim 60$ \\
\hline \multirow[t]{4}{*}{ Gent (1979) } & filter paper & $\mathrm{NaCl}$ & $\sim 45-85$ \\
\hline & & citric acid & $\sim 60-78$ \\
\hline & & quinine sulfate & $\sim 51-84$ \\
\hline & & sucrose & $\sim 57-74$ \\
\hline \multirow[t]{4}{*}{ Gent and McBurney (1978) } & filter paper & $\mathrm{NaCl}$ & $-75-90$ \\
\hline & & citric acid & $\sim 75-90$ \\
\hline & & quinine sulfate & $\sim 80$ \\
\hline & & sucrose & $\sim 70-85$ \\
\hline
\end{tabular}

Note-The degree of adaptation varies with concentration and stimulated area. 
r.p.m. for the $1,400-\mathrm{mPa} \cdot \mathrm{s}$ solutions, spindle 4 at 60 r.p.m. for the $4,200-\mathrm{mPa} \cdot \mathrm{s}$ solutions). Solutions were prepared at least 1 day before use and stored at $4^{\circ} \mathrm{C}$ for no more than 3 days. Before use, they were brought to room temperature $\left(23^{\circ} \mathrm{C}\right)$.

Design. The course of adaptation was established for three rates of mouth movements: no movement, standardized movement, and free movement. Each subject participated in three sessions. In each session, only one rate of mouth movements was employed. The six possible orders of mouth movements were used approximately equally often. In every session, each stimulus was judged five times, resulting in 30 stimuli per session. The order of these stimuli was randomized.

Procedure. The procedure was cued by a computer program providing sounds of moderate intensity. At a first bell-like signal, $5 \mathrm{ml}$ of the solution was pipetted on the tongue of the subject by the experimenter with an automatic pipette. The subject was asked to judge the sweetness intensity of the solution $2,5,15,25,35,45$, and $55 \mathrm{sec}$ after presentation of the stimulus. The judgments were expressed on a $150-\mathrm{mm}$ visual analogue scale anchored with not at all sweet on the left end and extremely sweet on the right end. Each judgment was made on a separate sheet. The moments at which the sweetness intensity had to be estimated were indicated by a $300-\mathrm{msec} 700-\mathrm{Hz}$ sound. After $55 \mathrm{sec}$, the solution was expectorated. The subject then rinsed his or her mouth twice with demineralized water and waited for the next stimulus. The intertrial interval was $50 \mathrm{sec}$. One session took about $1 \mathrm{~h}$.

In the standardized movement condition, the subjects were instructed to make mouth movements at a specified rate of 1.2 movements/sec. This rate of movement was indicated by $200-\mathrm{Hz}$ sounds of $40 \mathrm{msec}$. They were asked to keep the solution between the tongue and the palate with the lips closed and to make up- and downward mouth movements. They were told not to chew on the solutions. The experimenter demonstrated these movements. In the nomovement condition, the subjects were instructed to keep their mouth as still as possible. In the free movement condition, they were instructed to taste the solution as well as possible. This resulted in movements comparable with the standardized movements, at irregular time intervals.

Analysis. The distance from the left anchor of the visual analogue scale to the slash mark was measured in millimeters, providing a sweetness rating ranging between 0 (not at all sweet) and 150 (extremely sweet). In the analyses, the mean of the five replications was used.

The data were submitted to a repeated measures analysis of variance (ANOVA), with factors of concentration, rate of mouth movement, viscosity, and time (SPSS/PC+, Version 5.01). To test for differences in the degree of adaptation, the interaction of each factor with time was used. Multivariate tests of significance were used, if permitted by the number of degrees of freedom - that is, if the number of subjects was greater than the number of levels of repeated measures + 10 (Stevens, 1992, pp. 454-456). In all other cases, univariate tests were used. The degrees of freedom for the univariate tests were adjusted with the Huynh-Feldt epsilon (HFe), if the Greenhouse-Geisser epsilon (GGe) was larger than 0.7 , or with the mean of the GGe and the HFe, if the GGe was smaller than 0.7 (Stevens, 1992, pp. 454-456). These adjusted degrees of freedom are given between brackets.

In order to interpret possible differences with respect to the time course of adaptation, both linear and exponential functions were fitted to the mean group data, using the curve estimation procedure of SPSS 6.1 for Windows. This allows a comparison of the form of the adaptation curves and may help to explain differences between conditions. The degree of adaptation was estimated from these curves by subtracting the predicted response at $60 \mathrm{sec}$ from the predicted response at $0 \mathrm{sec}$ and dividing by the predicted response at $0 \mathrm{sec}$.

\section{Results}

Figure 1 shows the sweetness intensity estimates as a function of time for the three movement conditions, the two sucrose concentrations, and the three viscosity levels. Sweetness intensities do not differ for the different mouth movement conditions $[F(2,19)=1.58, p=.232]$. As was expected, the high concentration is judged sweeter than the low one $[F(1,20)=123.96, p<.001]$, and increasing viscosity is found to decrease sweetness intensity $[F(2,19)=35.96, p<.001]$.

Over all conditions, adaptation occurs $[F(6,15)=$ $8.09, p=.001]$. A differential effect on the degree of adaptation was only found for the factor of concentration $[F(1.8,36)=8.18, p<.01]$. As can be seen from Figure $1 \mathrm{~B}$, the low concentration shows a steeper decline of sweetness intensity than does the high concentration. However, this effect is apparent only during the first 5 or $15 \mathrm{sec}$. If the data from the measurement at $2 \mathrm{sec}$ are excluded from the analysis, the interaction is no longer significant $[F(1.6,31.7)=3.17, p>.05]$. Viscosity has no effect on the degree of adaptation $[F(2.2,43.2)=1.02$, $p>.05]$; neither has the mouth movement condition $[F(2.5,50.4)=0.75, p>.05]$.

To reveal the form of the adaptation curves, linear and exponential functions were fitted to the group data. Table 2 shows the regression coefficients and $R^{2}$. In almost all conditions, an exponential model showed the best fit, although the differences with the linear functions are small. The mean degree of adaptation over $60 \mathrm{sec}$, collapsed across concentration and viscosity levels and movement conditions, calculated from the fitted exponential functions is $\sim 33 \%$. For the low concentration, it is $\sim 42 \%$, whereas the high concentration shows $\sim 25 \%$ adaptation.

To examine whether individual subjects showed adaptation, we tested whether the sweetness judgment at $55 \mathrm{sec}$ was lower than the judgment at 2 sec with a Wilcoxon signed rank test ( $\alpha=.05$, one-tailed). Individual data for all concentration and viscosity levels were used. This test was performed only on the data from the no-movement condition, since this was the condition in which the highest degree of adaptation was expected. For 3 out of 21 subjects, this test was not significant, indicating that these subjects did not show adaptation.

\section{EXPERIMENT 2}

Unexpectedly, the mouth movement conditions in Experiment $l$ did not yield any differences in the time courses of adaptation. Because mouth movements cannot be ruled out in a no-movement sip-and-spit condition, the three conditions in Experiment 1 may not have been different enough to demonstrate an effect of mouth movements on taste adaptation. The purpose of the second experiment was to investigate whether more adaptation occurs when the stimulation method allows for less movement. We expected to find the highest degree of adaptation with 
sweetness

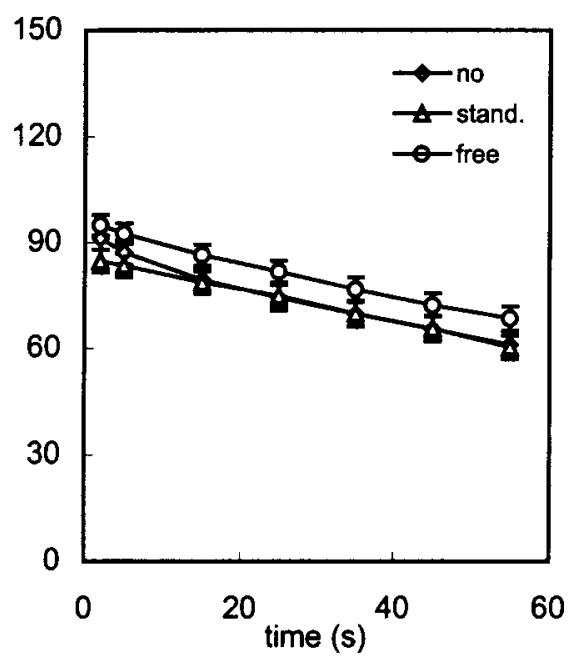

sweetness

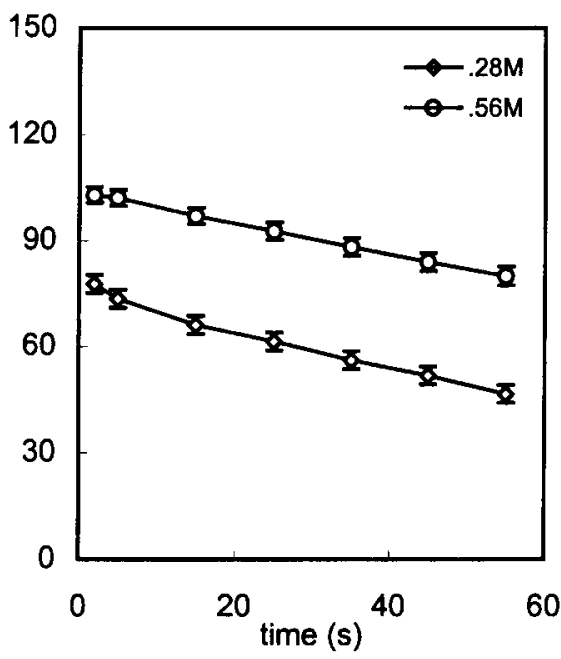

sweetness

C

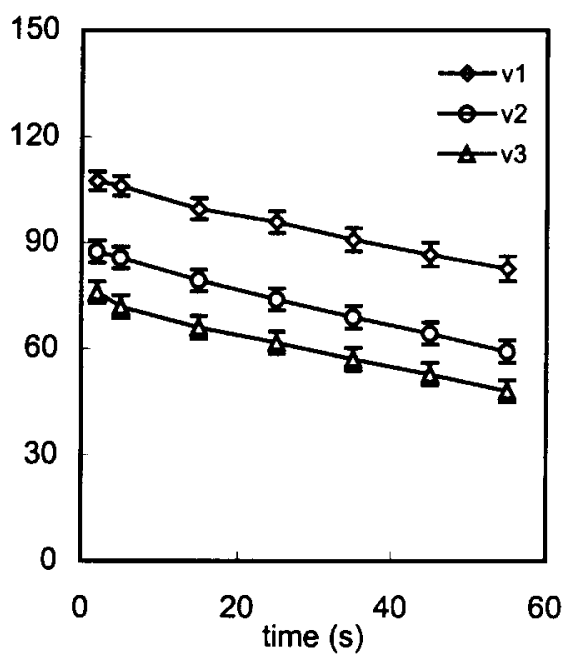

Figure 1. Sweetness intensity estimates as a function of time for the three mouth movement conditions (panel $A$ ), the two concentrations (panel $B$ ), and the three viscosity levels (panel $C$ ). V1 indicates solutions of $1 \mathrm{mPa} \cdot \mathrm{s} ; \mathrm{V} 2$ and $\mathrm{V} 3$ indicate the solutions of 1,400 and $4,200 \mathrm{mPa} \cdot \mathrm{s}$, respectively. Error bars indicate \pm 1 standard error of the mean.

the filter paper method and the lowest degree with the sip-and-spit method. The flow method was expected to lead to intermediate results.

\section{Method}

Subjects. Twenty-three paid volunteers, 17 females and 6 males, ranging in age from 18 to 40 years (median, 20 years), served as subjects. Most volunteers were students at Utrecht University. Some had experience with psychophysical experiments, but all were naive with respect to the purpose of the present experiment.

Stimuli. Sucrose (commercial grade, Suiker unie, Breda) was dissolved in demineralized water at least 1 day before use, and stored at $4^{\circ} \mathrm{C}$ for no more than 3 days. The stimuli were delivered at $34^{\circ} \mathrm{C}$. In the filter paper trials, circular pieces of filter paper (Whatman 91) with a diameter of $16 \mathrm{~mm}$ were used. These were soaked in solutions of 0.56 and $1.12 \mathrm{M}$ sucrose. In the flow and sipand-spit method 0.14 and $0.28 \mathrm{M}$ sucrose solutions were used. These concentrations were selected to yield approximately equal perceived sweetness intensities.

Design. All the subjects participated in three sessions. In each session, a different method of presentation was used: sip-and-spit, gravitational flow, or filter paper. The order of the presentation methods was balanced over subjects. In each session, both sucrose concentrations were tasted six times. The first 2 stimuli were always a high and a low concentration and were considered practice stimuli; these data were not included in the analyses. The next 10 stimuli were presented in a random order.

Procedure. The subjects first participated in a preliminary session. In this session, four stimuli (two high and two low concentrations) were presented using each presentation method. This was done in order to familiarize the subjects with the presentation methods 
Table 2

Regression Coefficients and $\boldsymbol{R}^{2}$ for Both Linear and Exponential Functions, Fitted to the Mean Group Data From Experiment 1

\begin{tabular}{|c|c|c|c|c|c|c|}
\hline \multirow[b]{2}{*}{ Condition } & \multicolumn{3}{|c|}{ Linear $\left(y=b_{0}+b_{1} x\right)$} & \multicolumn{3}{|c|}{ Exponential $\left(y=b_{0} e^{b 1 x}\right)$} \\
\hline & $b_{0}$ & $b_{1}$ & $R^{2}$ & $b_{0}$ & $b_{1}$ & $R^{2}$ \\
\hline No movement & 89.82 & -0.55 & .977 & 90.53 & -0.0073 & .990 \\
\hline Standardized movement & 85.90 & -0.46 & .998 & 86.59 & -0.0063 & .994 \\
\hline Free movement & 94.87 & -0.50 & .994 & 95.48 & -0.0061 & .999 \\
\hline $0.28 \mathrm{M}$ sucrose & 76.49 & -0.56 & .984 & 77.52 & -0.0092 & .995 \\
\hline $0.56 \mathrm{M}$ sucrose & 103.9 & -0.44 & .999 & 104.36 & -0.0048 & .999 \\
\hline Low viscosity & 107.85 & -0.47 & .994 & 108.32 & -0.0050 & .998 \\
\hline Medium viscosity & 87.886 & -0.53 & .997 & 88.71 & -0.0073 & .999 \\
\hline High viscosity & 74.849 & -0.50 & .988 & 75.68 & -0.0082 & .995 \\
\hline
\end{tabular}

and with the solutions used in the experiment. In the preliminary session, all the subjects started with the filter paper method, followed by the flow method, and ended with the sip-and-spit method.

The general procedure was cued by sound signals from a computer. At a $300-\mathrm{msec}$ sound of $900 \mathrm{~Hz}$, the stimulus was presented to the subject. After $5,10,15,25,35,45$, and $55 \mathrm{sec}$, the subject gave a sweetness estimate, cued by a $300-\mathrm{msec}$ sound of $700 \mathrm{~Hz}$. A $150-$ $\mathrm{mm}$ visual analogue scale was used, with the left end of the scale marked not at all sweet and the right end marked extremely sweet. Each judgment was made on a separate sheet. Once a judgment was made, the score sheet was removed.

In the filter paper session, the subjects were asked to extend their tongue between closed lips and dry it with filter paper (Whatman 91). The experimenter then soaked a piece of filter paper in the solution and put it on one side of the tongue, using a Teflon-coated forceps. Half of the subjects were stimulated on the right side of the tongue; the other half was stimulated on the left side. The stimulated tongue side was determined by the order of appearance: Subject 1 was stimulated on the left side, Subject 2 on the right side, and so forth. After $55 \mathrm{sec}$, the subjects removed the filter paper and rinsed their mouths thoroughly with demineralized water. In the flow session, a gravitational flow apparatus with one stimulus outlet was used to deliver the stimulus. The subjects were asked to ex- tend their tongue between closed lips, dry it with filter paper, and place it under a flow of $1.5 \mathrm{ml} / \mathrm{sec}$. No chinrest was employed. The stimulated area was about $200 \mathrm{~mm}^{2}$. After $55 \mathrm{sec}$, the flow was stopped, and the subjects rinsed their mouth thoroughly. In the sipand-spit session, the subjects were presented with a $5-\mathrm{ml}$ solution in a polystyrene medicine cup. They were told to take the solution in the mouth and keep the mouth motionless for the next $55 \mathrm{sec}$. Then they expectorated the solution and rinsed with demineralized water. In all sessions, the intertrial interval was at least $50 \mathrm{sec}$.

\section{Results}

We performed a repeated measures ANOVA comparable with the one in Experiment 1. Figure 2 shows the mean sweetness estimates for the three stimulation methods. For all the methods, higher concentrations yielded higher sweetness ratings $[F(1,22)=97.00, p<.001]$, and adaptation occurred $[F(6,17)=10.40, p<.001]$. Contrary to our findings in Experiment 1, concentration did not affect the degree of adaptation $[F(1.4,30.4)=$ $1.80, p>.05]$. Apparently, both concentrations adapted equally well. Stimulation method did affect the degree sweetness

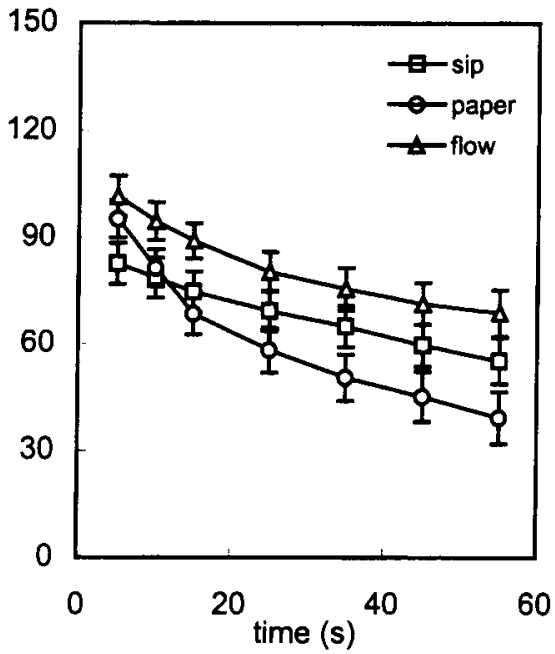

sweetness

B

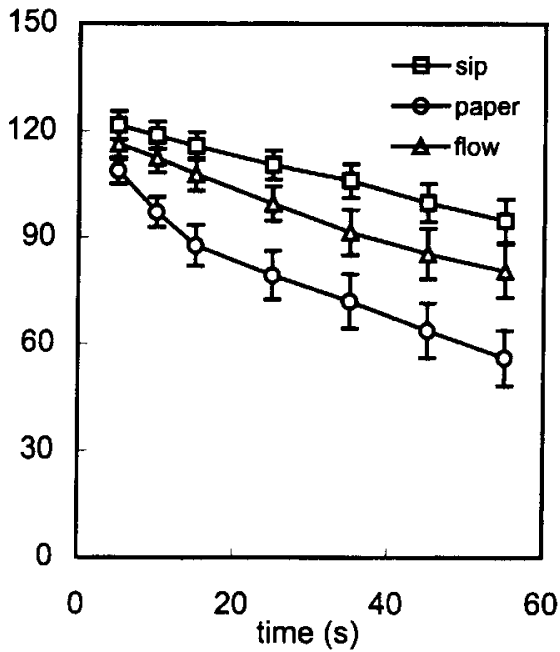

Figure 2. Sweetness intensity estimates as a function of time, with a separate curve for the three stimulation methods. Panel A shows the results for the low concentration, panel $B$ for the high concentration level. Each point is the mean of 115 judgments ( 23 subjects $\times 5$ replications). Error bars indicate \pm 1 standard error of the mean. 
Table 3

Regression Coefficients and $\boldsymbol{R}^{2}$ for Both Linear and Exponential Functions, Fitted to the Mean Group Data From Experiment 2

\begin{tabular}{lrrrrrrr}
\hline & \multicolumn{3}{c}{ Linear $\left(y=b_{0}+b_{1} x\right)$} & & \multicolumn{3}{c}{ Exponential $\left(y=b_{0} e^{b_{1} x}\right)$} \\
\cline { 2 - 4 } \cline { 7 - 8 } \multicolumn{1}{c}{ Condition } & \multicolumn{1}{c}{$b_{0}$} & \multicolumn{1}{c}{$b_{1}$} & $R^{2}$ & & \multicolumn{1}{c}{$b_{0}$} & \multicolumn{1}{c}{$b_{1}$} & $R^{2}$ \\
\hline Sip-and-spit, 0.28 M & 83.79 & -0.54 & .991 & & 85.01 & -0.0079 & .997 \\
Sip-and-spit, 0.56 M & 123.96 & -0.53 & .999 & & 124.77 & -0.0049 & .998 \\
Filter paper, 0.28 M & 90.66 & -1.03 & .906 & 94.60 & -0.0168 & .965 \\
Filter paper, 0.56 M & 107.14 & -0.98 & .958 & 110.43 & -0.0124 & .985 \\
Flow, 0.28 M & 100.43 & -0.64 & .936 & 101.47 & -0.0077 & .958 \\
Flow, 0.56 M & 118.98 & -0.74 & .990 & 120.54 & -0.0076 & .997 \\
\hline
\end{tabular}

of adaptation $[F(3.2,69.7)=8.67, p<.005]$. It can be seen from Figure 2 that, in the filter paper condition, more adaptation occurs than in both the sip-and-spit and the flow conditions. From repeated measures ANOVAs comparing two methods pairwise, these effects appear to be significant [paper vs. sip: $F(1.8,39.6)=16.94, p<$ .005 ; paper vs. flow: $F(1.7,37.6)=6.16, p<.01]$. The flow and the sip-and-spit conditions do not differ significantly $[F(1.3,29.4)=2.46, p>.05]$.

As in Experiment 1 , linear and exponential functions were fitted to the mean group data. The regression coefficients and $R^{2}$ are shown in Table 3. As in the first experiment, in almost all the conditions, the exponential model showed the best fit. The mean degree of adapta- tion over $60 \mathrm{sec}$, calculated from the fitted exponential functions, is $\sim 35 \%$ in the sip and the flow conditions, whereas in the filter paper condition, it is $\sim 58 \%$.

We tested whether individuals differed with respect to whether or not they showed adaptation in the three conditions. As in Experiment 1, a Wilcoxon signed rank test was used. In the sip-and-spit condition, 5 out of 23 subjects showed no adaptation. In both the filter paper and the flow conditions, 3 subjects did not show adaptation. To examine individual differences with respect to the degree of adaptation, the percentage of decline of sweetness intensity from 5 to $55 \mathrm{sec}$ was calculated. As was expected, there were large differences between subjects. The degrees of adaptation in the sip-and-spit condition of Experi-

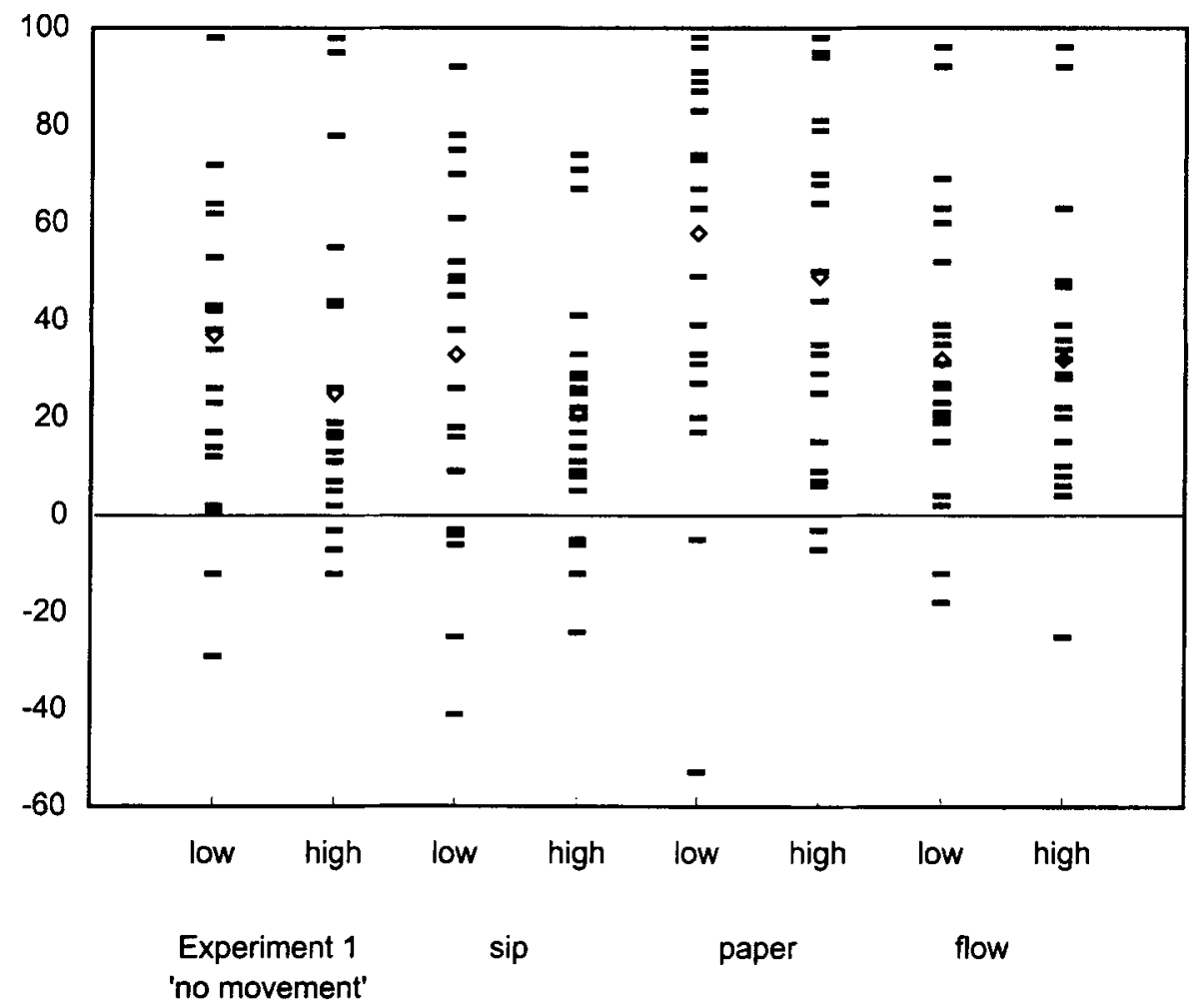

Figure 3. The mean individual degree of adaptation for each condition and concentration level in Experiment 2. Each horizontal line indicates the degree of adaptation of 1 person. Open diamonds show the group average. The results from the no-movement condition in Experiment 1, averaged over viscosity levels, are also shown. 
ment 2 are comparable with the values obtained in the no-movement condition in Experiment 1 (Figure 3).

\section{GENERAL DISCUSSION}

\section{Effect of Concentration on the Degree of Adaptation}

In Experiment 1, the low sucrose concentration appeared to adapt somewhat faster than the high concentration, whereas in Experiment 2, we found no effect of concentration. The reason for this discrepancy may be that the effect arises mainly during the first few seconds of stimulation. In Experiment 1, measurement started after $2 \mathrm{sec}$, whereas in Experiment 2, the first sweetness measurement was taken after $5 \mathrm{sec}$. This was done because the subjects administered the stimulus themselves in the sip-and-spit condition, and this gave them little time to give a judgment after $2 \mathrm{sec}$. However, a judgment at $5 \mathrm{sec}$ could be too late to observe the differential effect of concentration on the degree of adaptation. Furthermore, the effect of concentration on the degree of adaptation is small. This could explain why some previous studies found an effect of concentration on degree of adaptation (Gent, 1979), whereas others did not (Gent \& McBurney, 1978).

\section{Effect of Viscosity on the Degree of Adaptation}

Viscosity did not influence the degree of adaptation. Our reasoning was that, if viscosity lowers the effective concentration, it might also increase the degree of adaptation, similar to the effect of concentration. However, the differences in perceived sweetness intensity between the three viscosity levels are even smaller than the differences induced by concentration. A possible effect of viscosity on adaptation might be too small to be detected with a scaling method that is relatively insensitive to small differences. Our observation is in accordance with Gent and McBurney (1978), who note, in a small control experiment with 2 subjects, that no differences occurred in the time to complete adaptation for $\mathrm{NaCl}$ in water and $\mathrm{NaCl}$ in polyethylene glycol, a substance that increases solution viscosity.

\section{Effect of Mouth Movements and Method of Stimulation on the Degree of Adaptation}

In Experiment 1, we found no effect of mouth movements on the degree of adaptation. O'Mahony and Wong (1989) and Theunissen and Kroeze (1996), on the other hand, did find such an effect: Less adaptation occurred with than without mouth movements. These different results may be explained by individual differences with respect to the degree of adaptation. If large numbers of nonadapting subjects are included in the sample, mean or median results will show smaller differences in the degree of adaptation. This may have happened in the present Experiment 1.
In Experiment 2, the filter paper condition showed a significantly higher degree of adaptation than did the other conditions. The flow and the sip-and-spit conditions, either with or without mouth movements, did not differ with respect to the degree of adaptation. When a filter paper is used, hardly any movement of the stimulus with respect to the tongue is possible, whereas in the gravitational flow and in the sip-and-spit methods, tongue movements can shift the stimulus. Although the instructions in the no-movement conditions emphasized that the mouth should be kept completely still, several subjects reported that they found this hard to accomplish. Therefore, it is likely that, in all conditions except the filter paper condition, some movement of the stimulus through the mouth occurred. Probably, an extremely stable stimulus is needed to obtain a high degree of adaptation, and very slight mouth movements can already disrupt the adaptation process. More vigorous mouth movements may not decrease the degree of adaptation much further.

Even with the filter paper method, where mouth movements can hardly influence the stability of stimulation, 3 subjects showed no adaptation. Therefore, not only the ability to keep the mouth motionless, but also cognitive or physiological factors may affect the degree of adaptation. For instance, some subjects may be reluctant to give lower sweetness ratings for a stimulus that has not overtly changed, even though they experience a decreased sweetness intensity. This may be remedied by asking the subject to give only one judgment for each stimulus after a specific stimulus duration. Bujas, Szabo, Ajdukovic, and Mayer (1991) found a slightly steeper adaptation curve when 4 subjects gave only one intensity judgment than when they repeatedly gave intensity estimates. The disadvantage of this method is that it is time consuming, since it greatly increases the number of stimuli needed to estimate the time course of adaptation.

In most cases ( 11 out of 14 ; Tables 2 and 3 ), the exponential model gave a better fit to the time course of adaptation than did the linear model. The difference between the exponential and the linear models is small but is largest in the filter paper condition. An exponential decrease compares better with previous findings (Ganzevles \& Kroeze, 1987; Gent \& McBurney, 1978) and is in closer agreement with the course of receptor adaptation (Beidler, 1978; Bujas et al., 1991). Furthermore, the assumption that filter paper application reflects more closely the course of adaptation at receptor level fits with the finding that the distance between the exponential and the linear model is larger in the filter paper condition.

In conclusion, very slight mouth movements already reduce the degree of adaptation, whereas the stimulus characteristics of concentration and viscosity seem to have hardly any effect. During normal eating, numerous tongue, mouth, and jaw movements are made. Probably, these movements explain why taste intensity does not seem to disappear during meals. Also, people may not pay atten- 
tion to any decrease in intensity during eating, because they do not expect the taste of the food to change during eating. Using real foods instead of model stimuli is unlikely to influence the perceived degree of adaptation.

\section{REFERENCES}

BEIDLER, L. M. (1978). Biophysics and chemistry of taste. In E. C. Carterette \& M. P. Friedman (Eds.), Handbook of perception: Vol. VIA. Tasting and smelling (pp. 21-49). New York: Academic Press.

Bujas, Z., Szabo, S., Ajdukovic, D., \& Mayer, D. (1991). Magnitude estimation of adaptation to salt using a flow chamber for stimulus delivery. Physiology \& Behavior, 49, 735-737.

Christensen, C. M. (1980). Effects of solution viscosity on perceived saltiness and sweetness. Perception \& Psychophysics, 28, 347-353.

DuBose, C. N., \& Meiselman, H. L. (1979). Individual differences in taste adaptation. Chemical Senses \& Flavor, 1, 177-181.

DuBose, C. N., Meiselman, H. L., Hunt, D. A., \& Waterman, D. (1977). Incomplete taste adaptation to different concentrations of salt and sugar solutions. Perception \& Psychophysics, 21, 183-186.

Ganzevles, P. G. J., \& Kroeze, J. H. A. (1987). Cross-adaptation in taste measured with a filter paper method. Chemical Senses, 12, 341-353.

Gent, J. F. (1979). An exponential model for adaptation in taste. Sensory Processes, 3, 303-316

Gent, J. F., \& McBurney, D. H. (1978). Time course of gustatory adaptation. Perception \& Psychophysics, 23, 171-175.

HAHN, H. (1934). Die adaptation des Geschmacksinnes [Taste adaptation]. Zeitschrift für Sinnesphysiologie, 65, 105-145.

LAWless, H. T., \& SkINNER, E. Z. (1979). The duration and perceived intensity of sucrose taste. Perception \& Psychophysics, 25, 180-184.

MCBURNEY, D. H. (1985). Taste and olfaction: Sensory discrimination. In J. M. Brookhart \& V. B. Mountcastle (Eds.), Handbook of physi- ology: Section I, Vol. II. Sensory Processes (pp. 1067-1086). Bethesda, MD: American Physiological Society.

Meiselman, H. L. (1968). Magnitude estimations of the course of gustatory adaptation. Perception \& Psychophysics, 4, 193-196.

Meiselman, H. L., \& Buffington, C. (1980). Effects of filter paper stimulus application on gustatory adaptation. Chemical Senses, 5, 273-277.

O'MAHONY, M. (1986). Sensory adaptation. Journal of Sensory Studies, 1, 237-258.

O'Mahony, M., \& Wong, S. Y. (1989). Time-intensity scaling with judges trained to use a calibrated scale: Adaptation, salty and umami tastes. Journal of Sensory Studies, 3, 217-236.

Schiffman, S. S., Pecore, S. D., Booth, B. J., Losee, M. L., Carr, B. T., Sattely-Miller, E., Graham, B. G., \& Warwick, Z. S. (1994). Adaptation of sweeteners in water and in tannic acid solutions. Physiology \& Behavior, 55, 547.559.

Smith, D. V., Bealer, S. L., \& VAN Buskirk, R. L. (1978). Adaptation and recovery of the rat chorda tympani response to $\mathrm{NaCl}$. Physiology \& Behavior, 20, 629-636.

STEVENS, J. (1992). Applied multivariate statistics for the social sciences. Hillsdale, NJ: Erlbaum.

Theunissen, M. J. M., \& Kroeze, J. H. A. (1996). Mouth movements diminish taste adaptation, but rate of mouth movement does not affect adaptation. Chemical Senses, 21, 545-551.

VON BÉKÉsY, G. (1965). The effect of adaptation on the taste threshold observed with a semiautomatic gustometer. Journal of General Physiology, 48, 481-488.

ZOTTERMAN, Y. (1971). The recording of the electrical response from human taste nerves. In E. M. Beidler (Ed.), Handbook of sensory physiology: Vol IV. Chemical senses: Part 2. Taste (pp. 102-115). Berlin: Springer-Verlag.

(Manuscript received March 4, 1998; revision accepted for publication January $5,1999$. 